\title{
Microwave-assisted synthesis of 3-arylcoumarins
}

\author{
Julio A. Seijas,* M. Pilar Vázquez-Tato,* José Crecente-Campo
}

Departamento de Química Orgánica. Facultade de Ciencias. Universidade de Santiago de Compostela. Campus de Lugo. Alfonso X el Sabio, 27002-Lugo. Spain.

julioa.seijas@usc.es, pilar.vazquez.tato@usc.es

Abstract: 3-arylcoumarins are prepared in good yields by microwave assisted solvent-free condensation of phenylacetic acids and phenylacetic esters, using potassium tert-butoxide as base.

The 3-arylcoumarins are closely related to the flavonoids. The most studied 3-phenylcoumarin is coumestrol, in fact, it gives name to a whole family of compounds (coumestanes) with important biological properties. Coumestrol is considered one of the most important phytoestrogens, along with isoflavones and lignans. Phytoestrogens have attracted much attention in recent years as they have beneficial properties in diseases of great impact on our day, such as cancer, heart disease, osteoporosis and menopausal symptoms. Natural or synthetic 3arylcoumarins have significant biological properties, such as inhibitors of various types of peroxidise, ${ }^{1}$ enzymes in the glycolitic path of Trypanosoma cruzi ${ }^{2}$ being potential anti-protozoos ${ }^{3}$ and moderate antifungic activity. ${ }^{4}$ There are also 3arylcoumarins that inhibit the oxidative metabolism of neutrophils stimulated by immune response. ${ }^{5}$

Continuing with our studies on microwave assisted organic synthesis, ${ }^{6-16}$ we draw our attention to this kind of compounds since there are only two previous reports on the use of microwaves to prepare 3-arylcoumarins. Wei et al. ${ }^{17}$ condensate phenylacetic acids and salicylaldehyde derivatives in the presence of $\mathrm{Et}_{3} \mathrm{~N}$ and $\mathrm{Ac}_{2} \mathrm{O}$. Bogdal ${ }^{18}$ prepared a 3-arylcoumarin by Knoevenagel condensation using piperidine as base.

Due to our own experience in knoevenagel condensations assisted with microwaves using potassium tert-butoxide, we investigated the application of these conditions to the synthesis of 3-arylcoumarins. As a model, the synthesis of 3phenylcoumarin from ethyl phenylacetate and salicylaldehyde was chosen (scheme 1). ${ }^{19}$ Different sets of conditions (reaction times, temperatures and reagent molar ratio of ethyl phenylacetate, salicylaldehyde and potassium tert-butoxide) were tried (table 1), finding the best yield (91\%) for a molar ratio $\mathrm{PhCH}_{2} \mathrm{CO}_{2} \mathrm{Et}$ : salicylaldehyde: t-BuOK 1:2:0.5, irradiating with $300 \mathrm{~W}$ power at $100^{\circ} \mathrm{C}$ and 
simultaneous cooling (table 1 , entry 9). Longer irradiation times rendered lower yield of 3-arylcoumarin ( $85 \%$, table 1 , entry 7 ).

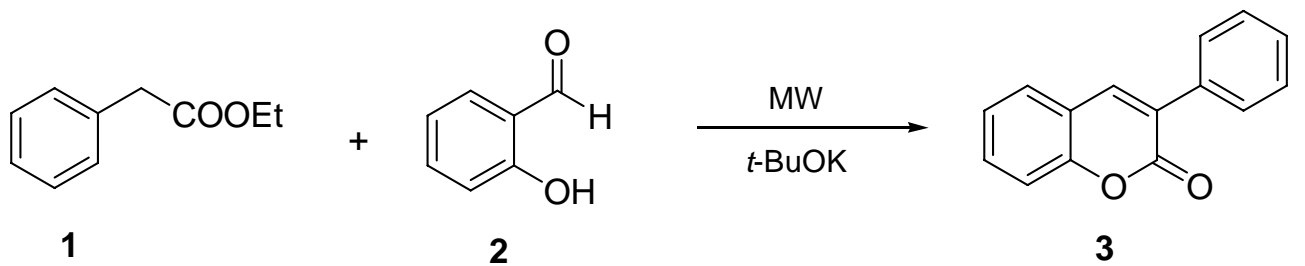

Scheme 1

Table 1

\begin{tabular}{lllll}
\hline Entry & Molar ratio 1:2:t-BuOK & Irradiation time $(\mathrm{min})$ & $\mathrm{T}\left({ }^{\circ} \mathrm{C}\right)$ & Yield of $\mathbf{3}(\%)$ \\
\hline 1 & $1: 1: 0.1$ & 10 & 180 & 24 \\
2 & $1: 1: 0.1$ & 3 & 100 & 18 \\
3 & $1: 1: 0.1$ & 20 & 100 & 50 \\
4 & $1: 1: 0.4$ & 30 & 100 & 50 \\
5 & $1.5: 1: 0.4$ & 30 & 100 & 79 \\
6 & $1.5: 1: 1$ & 30 & 100 & 60 \\
7 & $2: 1: 0.5$ & 45 & $100\left(P_{\max }{ }^{a}\right)$ & 85 \\
8 & $2: 1: 0.5$ & 20 & $80\left(P_{\max }{ }^{a}\right)$ & 10 \\
9 & $2: 1: 0.5$ & 15 & $100\left(P_{\max }{ }^{a}\right)$ & 91
\end{tabular}

${ }^{a} \mathrm{P}_{\max }=$ Power maximum (irradiation with simultaneous cooling with pressured air at room temperature of the irradiation chamber in order to maximize the absorption of microwaves).

Phenylacetic acids instead of their esters were tried (scheme 2). So, 3-(2hydroxyphenyl)-2H-chromen-2-one (5) and 3-(benzo[d][1,3]dioxol-5-yl)-2Hchromen-2-one (6) were prepared, in very good yields, from 2-(2hydroxyphenyl)acetic acid (4a) and 3,4-(methylenedioxy)phenylacetic acid (4b), respectively, using the same conditions described above (table 2, entries 2 and 4).<smiles>O=c1oc2ccccc2cc1-c1ccccc1O</smiles>

5<smiles>CC(C)(C)OCc1ccc(C=O)c(O)c1</smiles>

2<smiles>Cc1ccc(CC(=O)O)cc1C[13C](C)(C)O[13C](C)(C)C</smiles>

6

Scheme 2

Table 2

\begin{tabular}{lllll}
\hline Entry & Molar ratio 4:2:t-BuOK & Irr. time $(\mathrm{min})$ & $\mathrm{T}\left({ }^{\circ} \mathrm{C}\right)$ & Compound (yield, \%) \\
\hline \hline 1 & $1.5: 1: 0.5$ & 15 & $100\left(\mathrm{P}_{\max }{ }^{\mathrm{a}}\right)$ & $\mathbf{5}(77)$ \\
2 & $2: 1: 0.5$ & 15 & $100\left(\mathrm{P}_{\max }{ }^{\mathrm{a}}\right)$ & $\mathbf{5}(88)$ \\
3 & $1.5: 1: 0.5$ & 15 & $100\left(\mathrm{P}_{\max }{ }^{\mathrm{a}}\right)$ & $\mathbf{6}(63)$ \\
4 & $2: 1: 0.5$ & 15 & $100\left(\mathrm{P}_{\max }{ }^{\mathrm{a}}\right)$ & $\mathbf{6}(87)$ \\
\hline
\end{tabular}

${ }^{\mathrm{a}} \mathrm{P}_{\max }=$ Power maximum 
In summary, a microwave-assisted direct synthesis for 3-arylcoumarins under solventless conditions, catalyzed with potassium tert-butoxide is reported. The examples studied promise a wide field of application, which is still under study.

\section{General procedure:}

In a special glass tube for microwave oven (CEM, Discover) were mixed thoroughly phenylacetic acid or its ethyl ester $(2 \mathrm{mmol})$, salicylaldehyde $(1 \mathrm{mmol})$ and t-BuOK $(0.5 \mathrm{mmol})$. The mixture was irradiated using a power of $300 \mathrm{~W}$, for 15 minutes at $100^{\circ} \mathrm{C}$, with continuous sample cooling by means of pressured air at room temperature. The reaction was suspended in dichloromethane and the resulting solid filtered off. The organic solution was evaporated and the residue additionally purified by chromatography.

Acknowledgement We acknowledge XUNTA DE GALICIA for financial support: PGIDIT05PXIB26201PR, PR405 A098/59-0. USC for a predoctoral fellowship to J. C.-C. This work was undertaken as part of the EC sponsored programs D32 COST Program (Chemistry in High-Energy Microenvironments, WG10).

\section{References and notes}

1. Kabeya, L. M.; De Marchi, A. A.; Kanashiro, A.; Lopes, N. P.; Da Silva, C. H. T. P.; Pupo, M. T.; Lucisano-Valim, Y. M. Bioorg. Med. Chem. 2007, 15, 15161524.

2. De Marchi, A. A.; Castilho, M. S.; Nascimento, P. G. B.; Archanjo, F. C.; Del Ponte, G.; Oliva, G.; Pupo, M. T. Bioorg. Med. Chem. 2004, 12, 4823-4833.

3. Singh, S.; Kishen-Malik, B.; Kumar-Sharma, D. Chem. Biol. Drug Des. 2008, 71, 554-562.

4. Arnoldi, A.; Farina, G.; Galli, R.; Merlini, L.; Grazia-Parrino, M. J. Agric. Food Chem. 1986, 34, 185-188.

5. Kabeya, L. M.; Da Silva, C. H. T. P.; Kanashiro, A.; Campos, J. M.; Azzolini, A. E. C. S.; Polizello, A. C. M.; Pupo, M. T.; Lucisano-Valim, Y. M. Eur. J. Med. Chem. 2008, 3, 996-1007.

6. Vázquez-Tato, M. P. Synlett 1993, 506.

7. Oliveira-Campos, A. M. F.; Sivasubramanian, A.; Rodrigues, L. M.; Seijas, J. A.; Vázquez-Tato, M. P.; Peixoto, F.; Abreu , C. G.; Cidade, H.; Oliveira, A. E.; Pinto, M. Helvetica Chimica Acta 2008, 91, 1336-1345.

8. Seijas, J. A.; Vázquez-Tato, M. P.; Crecente-Campo, J. Tetrahedron 2008, 64, 9280-9285.

9. Seijas, J. A.; Vázquez-Tato, M. P.; Carballido-Reboredo, M. R. J. Org. Chem. 2005, 70, 2855-2858. 
10. Seijas, J. A.; Vázquez-Tato, M. P.; Martínez, M. M.; Rodríguez-Parga, J. Green Chem. 2002, 390-391.

11. Seijas, J. A.; Vázquez-Tato, M. P.; Martínez, M. M. Synlett 2001, 875-877.

12. Seijas, J. A.; Vázquez-Tato, M. P.; González-Bande, C.; Martínez, M. M.; LópezPacios, B. Synthesis 2001, 999-1000.

13. Seijas, J. A.; Vázquez-Tato, M. P.; Martínez, M. M. Tetrahedron Lett. 2000, 41, 2215-2217.

14. Seijas, J. A.; Vázquez-Tato, M. P.; Martínez, M. M.; Núńez-Corredoira, G. J . Chem. Res. (S) 1999, 420-421.

15. Seijas, J. A.; Vázquez-Tato, M. P.; Carballido-Reboredo, M. R.; CrecenteCampo, J.; Romar-López, L. SynLett 2007, 313-317.

16. Seijas, J. A.; Vázquez-Tato, M. P.; Crecente-Campo, J. Synlett 2007, 24202424.

17. Wei, J.; Ma, W.-H.; Xu, Q. J ingxi Yu Zhuanyong Huaxuepin 2006, 14, 8-12. Chemical Abstracts CAN 148:285003.

18. Bogdal D. J. Chem. Research (S) 1998, 468-469.

19. To our knowledge no previous synthesis for 3-phenylcoumarin has been previously described using these two starting materials. 